\title{
Alterações cognitivas na esquizofrenia: atualização
}

\author{
Cognitive impairment in schizophrenia: an update
}

\author{
Breno de Castro Ferreira Junior ${ }^{1}$, Marilourdes do Amaral Barbosa ${ }^{2}$, Izabela Guimarães Barbosa ${ }^{3}$, Cláudia \\ Hara $^{4}$, Fábio Lopes Rocha ${ }^{5}$
}

${ }^{1}$ MD, MSc. Coordenador, Serviço de Interconsulta, Clínica Psiquiátrica, Instituto de Previdência dos Servidores do Estado de Minas Gerais (IPSEMG), Belo Horizonte, MG. ${ }^{2}$ MSc. Psicóloga. Professora, Universidade Fundação Mineira de Educação e Cultura (FUMEC), Belo Horizonte, MG. ${ }^{3}$ MD, MSc. Psiquiatra, IPSEMG. ${ }^{4}$ MD, PhD. Professora, Faculdade de Saúde e Ecologia Humana (FASEH), Vespasiano, MG. ${ }^{5}$ MD, PhD. Coordenador, Pós-Graduação e Clínica Psiquiátrica, IPSEMG. O presente estudo foi realizado no Instituto de Previdência dos Servidores do Estado de Minas Gerais (IPSEMG), Belo Horizonte, MG.

\section{Resumo}

As alterações cognitivas são características centrais na esquizofrenia. Elas permanecem relativamente estáveis durante todo o curso da doença, não sendo secundárias a outros sintomas ou a efeitos colaterais de psicofármacos. Estão diretamente ligadas a prejuízo funcional e a pior qualidade de vida dos pacientes. Diversos estudos vêm sendo realizados no sentido de caracterizar as principais alterações cognitivas na esquizofrenia, identificar suas bases neurobiológicas e padronizar instrumentos de pesquisa, fundamentais para o advento de novos alvos para intervenções farmacológicas na esquizofrenia. O objetivo deste trabalho foi fazer uma atualização sobre o assunto.

Descritores: Esquizofrenia, cognição, avaliação, escalas.

\begin{abstract}
Cognitive dysfunctions are among the core features of schizophrenia. They remain relatively stable throughout the course of the disease, and are secondary neither to other symptoms nor to side effects of psychotropic drugs. Cognitive deficits are directly associated with functional impairment and poor quality of life. Many studies have been conducted to describe the main cognitive abnormalities observed in schizophrenia, to identify their neurobiological bases, and to standardize research instruments, which are of paramount importance for the identification of new targets for pharmacological interventions in schizophrenia. The main objective of this paper was to provide an updated review of the subject.
\end{abstract}

Keywords: Schizophrenia, cognition, evaluation, scales.

\section{Introdução}

Prejuízos cognitivos são alterações primárias da esquizofrenia. Historicamente, Emil Kraepelin, na primeira sistematização conceitual da esquizofrenia, em 1896, descreveu diversos sintomas cognitivos na demência precoce (distúrbios da atenção, da compreensão, do fluxo e associação do pensamento e alterações do julgamento), assim como também sua evolução para a deterioração cognitiva e da personalidade. Eugen Bleuler, em 1911, considerou a avolição como uma característica central da doença. Esse sintoma está associado a déficits executivos relacionados à disfunção do lobo frontal ${ }^{1}$. Apesar de a disfunção cognitiva não ser um critério de inclusão para o diagnóstico de esquizofrenia nas classificações atuais, a conceituação atual menciona as alterações cognitivas entre os sintomas característicos da doença $a^{1,2}$.
As alterações cognitivas na esquizofrenia são evidentes durante todo o curso evolutivo da doença, inclusive no seu período prodrômico. Essas alterações não são secundárias a outros sintomas e não são resultantes de tratamentos com psicofármacos ou de hospitalizações ${ }^{3,4}$. Também são identificáveis, com menor gravidade, nos pais e irmãos saudáveis dos pacientes ${ }^{5}$. Estudos têm sido realizados buscando esclarecer quais seriam os déficits cognitivos específicos da esquizofrenia, suas ligações com disfunções de regiões cerebrais e certos circuitos neuronais, suas repercussões sobre a funcionalidade dos pacientes e as possibilidades terapêuticas'.

O objetivo deste estudo foi apresentar, de forma didática, as alterações cognitivas da esquizofrenia, suas bases e suas perspectivas terapêuticas, através de uma atualização crítica da literatura. Foi realizada uma revisão não sistemática da literatura indexada nas bases de dados MEDLINE, LILACS e

Correspondência:

Breno de Castro Ferreira Junior, Rua Domingos Vieira, 587, CEP 30150-240, Belo Horizonte, MG. E-mail: brenoferreirajr@hotmail.com

Não foram encontrados conflitos de interesse associados à publicação deste artigo.

Copyright (C) Revista de Psiquiatria do Rio Grande do Sul - APRS

Recebido em 17/01/2010. Aceito em 09/03/2010. 
Biblioteca Cochrane, no período de janeiro de 2004 a dezembro de 2009. Os descritores utilizados foram schizophrenia, cognition, cognitive assessment, cognitive impairment, cognitive impairment treatment, adjunctive therapy e executive function. Os artigos selecionados foram analisados e comparados. Também foram utilizados livros-textos e artigos selecionados a partir de citações em outros artigos e livros-textos de neuropsicologia e psicologia.

\section{A inteligência: modelo Cattell-Horn-Carroll (CHC)}

Para a compreensão das alterações cognitivas na esquizofrenia, será apresentado e discutido, em primeiro lugar, o modelo psicológico da inteligência. Esse modelo possibilita a compreensão das diversas funções cognitivas de forma integrada e orgânica. Permite também uma melhor compreensão do alcance e limitações das avaliações neuropsicológicas atuais. $\mathrm{O}$ conceito atual de inteligência pode ser representado pela teoria das capacidades cognitivas de Cattell-Horn-Carroll (teoria do modelo CHC). Segundo esse modelo, a inteligência é o resultado de capacidades múltiplas, estimuláveis e passíveis de testagem ${ }^{7}$, sendo estruturada em três camadas. O fator $g$ (inteligência geral), situado na camada III, é constituído por 10 fatores cognitivos gerais, situados na camada II. Esses fatores são: 1) inteligência/raciocínio fluido (Gf); 2) raciocínio/conhecimento quantitativo $(\mathrm{Gq}) ; 3)$ inteligência/raciocínio cristalizado (Gc); 4) leitura e escrita (Grw); 5) memória de curto prazo (Gsm); 6) processamento visual (Gv); 7) processamento auditivo (Ga); 8) armazenamento e recuperação a longo prazo (Glr); 9) inteligência/velocidade de processamento (Gs); e 10) rapidez de decisão (Gt). Na camada I estão mais de 70 habilidades/fatores específicos que se inter-relacionam e se agrupam de vários modos, originando cada um dos 10 fatores gerais (Figura 1$)^{8}$.

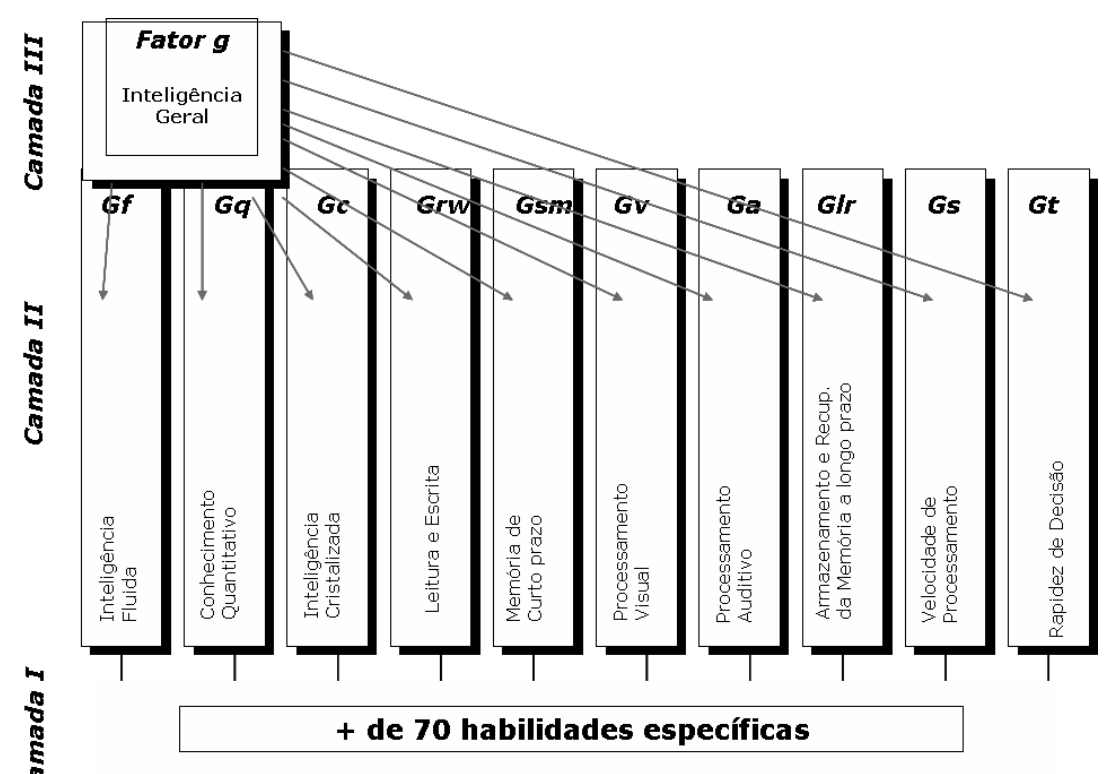

Figura 1 - Cattell-Horn-Carroll (CHC) (adaptado de $\mathrm{McGrew}^{8}$ )

Assim, ao se avaliar um dos fatores cognitivos gerais da camada II - por exemplo, a Leitura -, na realidade, várias habilidades específicas da camada I estão sendo apreciadas em conjunto, não sendo possível precisar comprometimentos específicos.

Por outro lado, a investigação de habilidades ou fatores específicos da inteligência, localizados na camada I, não permite inferir adequadamente o impacto de uma disfunção específica sobre os fatores cognitivos gerais e, menos ainda, seu impacto no desempenho global e funcionalidade do indivíduo. Sendo assim, o resultado de testes cognitivos muitas vezes não permite inferências precisas acerca do funcionamento do indivíduo no dia a dia. Esse modelo integrado de inteligência também ajuda a compreender a grande dificuldade em se estabelecer correlações entre os resultados de avaliações cognitivas e eventuais substratos neurofuncionais específicos ${ }^{9}$. Na esquizofrenia, as avaliações neurocognitivas mostram alterações de habilidades ou fatores específicos da inteligência (camada I), os quais, potencialmente, podem comprometer diversos fatores cognitivos gerais (camada II) e o funcionamento cotidiano do paciente.

\section{Avaliação das alterações cognitivas}

As pesquisas iniciais acerca das alterações cognitivas na esquizofrenia caracterizaram-se pela grande diversidade de métodos utilizados, com prejuízo para a interpretação e comparação dos resultados dos diversos estudos. Em 2005, Fioravanti et al., em uma meta-análise de 113 estudos pu- 
blicados entre 1990 e 2003, envolvendo um total de 4.365 pacientes e 3.429 controles saudáveis, mostrou a necessidade de métodos específicos e padronizados para a testagem cognitiva desses pacientes ${ }^{10}$.

Em resposta a essa necessidade e tendo como uma das metas a pesquisa de terapêuticas para as disfunções cogniti- vas da esquizofrenia, o National Institute of Mental Health (NIMH), nos EUA, instituiu o programa Measurement and Treatment Research to Improve Cognition in Schizophrenia (MATRICS $)^{11}$. O MATRICS padronizou uma bateria de testes para a avaliação das disfunções cognitivas na esquizofrenia, com boa sensibilidade e especificidade (Tabela 1) ${ }^{12,13}$.

Tabela 1 - Bateria de testes padronizada para a avaliação de disfunções cognitivas na esquizofrenia (MATRICS) (adaptado de Buchanan et al. ${ }^{13}$ )

\begin{tabular}{ll}
\hline Domínio cognitivo & Teste \\
\hline Velocidade de processamento & $\begin{array}{l}\text { Category Fluency } \\
\text { Brief Assessment of Cognition in Schizophrenia (BACS) } \\
\text { Symbol-Coding Trail Making A }\end{array}$ \\
& $\begin{array}{l}\text { Continuous Performance Test, Identical Pairs (CPT-IP) } \\
\text { Atenção/vigilância }\end{array}$ \\
& Nerbal: University of Maryland, Letter/Number Span \\
Aprendizado verbal & Hopkins Verbal Learning Test (HVLT) - Revised \\
Aprendizado visual & Brief Visospatial Memory Test (BVMT) - Revised \\
Capacidade de resolução de problemas & Neuropsychological Assessment Battery (NAB), Mazes \\
Cognição social & Mayer-Salovey-Caruso Emotional Intelligence Test (MSCEIT) \\
\end{tabular}

No entanto, a bateria do MATRICS tem limitações. É muito extensa, pouco prática e dispendiosa em termos de tempo e custo. Além disso, exige conhecimentos especializados para a sua aplicação e interpretação, o que inviabiliza sua utilização em diversos serviços e, principalmente, na rotina clínica. Outras baterias foram desenvolvidas com o objetivo de ser breves e de fácil administração. Uma dessas baterias encontra-se validada no Brasil: a Brief Assessment of Cognition in Schizophrenia (BACS) ${ }^{14}$, que cobre a maioria dos déficits cognitivos da esquizofrenia. Os domínios cognitivos avaliados são memória verbal, memória de trabalho, velocidade motora, atenção, solução de problemas e fluência verbal.

Outra limitação das baterias tradicionais se relaciona à discussão apresentada no tópico anterior, sobre a teoria das capacidades cognitivas. Trata-se da dificuldade em extrapolar o resultado da bateria de testes para o desempenho do paciente em suas atividades do dia a dia. Testes têm sido desenvolvidos com a finalidade de sobrepujar essas limitações. São avaliações baseadas em entrevistas relativamente curtas, com a meta de investigar a funcionalidade do paciente para as atividades diárias. Um desses testes, a Schizophrenia Cognition Rating Scale (SCoRS), tem se mostrado prático e apresentado boa correlação entre a capacidade funcional medida e as capacidades cognitivas desses pacientes ${ }^{15}$. A SCoRS foi traduzida para o português para validação no Brasil por um dos autores (F.L.R.), com a tradução reversa avaliada pelo autor da escala (resultados aguardando publicação). Para uma síntese acerca dos estudos sobre os testes neuropsicológicos mais utilizados em esquizofrenia recomenda-se a revisão sistemática de Zimmer et al. ${ }^{16}$.

\section{Principais alterações cognitivas na esquizofrenia}

As principais alterações cognitivas da esquizofrenia foram sintetizadas em uma meta-análise envolvendo 13 estudos realizados com metodologia adequada, que permitiam a comparação dos resultados ${ }^{17}$ :

- Velocidade de processamento: habilidade associada à taxa de rapidez do processamento cognitivo em tarefas simples (por exemplo: nomear cores);

- Atenção/vigilância: habilidade de manter a atenção voltada para uma tarefa (foco ao longo do tempo) (por exemplo: assistir a um filme na televisão);

- Memória de trabalho (working memory): habilidade de reter certa quantidade de informações para utilização imediata (por exemplo: dizer se uma sequência de letras apresentada é igual a uma sequência anterior);

- Aprendizado verbal e memória: habilidade de aprender informações verbalizadas e se recordar delas (memória imediata preservada e memória de longo prazo/reconhecimento deficitária);

- Aprendizado visual e memória: habilidade de gerar, reter e manipular imagens visuais (associado aos diferentes aspectos de processamento de imagens, como geração, transformação, armazenamento e recuperação);

- Raciocínio/solução de problemas: habilidade de raciocínio em situações novas, minimamente dependentes de conhecimentos adquiridos (capacidade de resolver problemas novos, associar ideias, extrapolar e reorganizar informações); 
- Cognição social: habilidade relacionada à capacidade de perceber e compreender as regras sociais (capacidade de estabelecer relações sociais).

Essas alterações cognitivas correspondem a alterações nas camadas I e II da teoria CHC da inteligência, exceto a cognição social, que é um aspecto cognitivo especializado e desenvolvido pelo indivíduo com o objetivo de resolver problemas para sua adequada adaptação social. $\mathrm{O}$ córtex pré-frontal parece estar envolvido no desenvolvimento dessas habilidades. Nos indivíduos sadios, processos como empatia e julgamentos de base ética e moral estão associados à cognição social. No paciente com esquizofrenia, o comprometimento da cognição social é considerado o fator responsável pelo isolamento social do paciente e está presente desde o primeiro episódio psicótico $^{18}$. Na teoria $\mathrm{CHC}$ da inteligência, a cognição social não é contemplada, uma vez que, em psicologia, esse aspecto é tratado no contexto das teorias da personalidade.

\section{Características das disfunções cognitivas da esquizofrenia}

As alterações cognitivas da esquizofrenia têm elevada prevalência, são contínuas e apresentam elevada gravidade. Estudos comparando o desempenho de pacientes esquizofrênicos com controles saudáveis em baterias de testes cognitivos mostraram distribuição normal dos resultados para os dois grupos, mas com grande parte dos pacientes tendo desempenho pelo menos 1,5 desvio padrão abaixo da média dos controles saudáveis. Considerando o critério de 1 desvio padrão como ponto de corte, $80 \%$ dos pacientes incluídos apresentavam alterações cognitivas ${ }^{4,19}$.

Com relação ao curso da doença, estão bem estabelecidas a precocidade do surgimento das alterações cognitivas na esquizofrenia e sua estabilidade em longo $\mathrm{prazo}^{2}$. Essas alterações podem estar presentes mesmo antes do primeiro surto psicótico. Por exemplo, Cannon et al., em 2002, encontraram pior desempenho escolar pré-mórbido em esquizofrênicos quando comparados a controles saudáveis e a portadores de doenças afetivas ${ }^{20}$. Keefe et al., em 2007, demonstraram o agravo de dificuldades cognitivas durante a adolescência em indivíduos que apresentavam risco para o desenvolvimento da doença ${ }^{4}$. Também foi demonstrado que a gravidade das alterações cognitivas em pacientes com esquizofrenia está associada a mau desempenho escolar pré-mórbido ${ }^{21}$. Questiona-se, atualmente, se essas alterações cognitivas prodrômicas, juntamente com outros fatores de risco para esquizofrenia e outras psicoses, poderiam constituir uma nova categoria diagnóstica na $5^{\mathrm{a}}$ edição do Diagnostic and Statistical Manual of Mental Disorders (DSM-V) ${ }^{22}$. O prejuízo intelectivo e o desempenho escolar inferior, presentes antes do surgimento da doença, acentuam-se nos 3 a 5 primeiros anos após o início da doença e, em seguida, permanecem relativamente estáveis ao longo do curso da enfermidade ${ }^{20}$.
Em relação à gravidade, vários estudos mostram que as alterações cognitivas da esquizofrenia são mais acentuadas quando comparadas às alterações das doenças afetivas ${ }^{3,23-25}$. Por exemplo, em pacientes acompanhados por 2 anos após a primeira manifestação da doença, aqueles diagnosticados com esquizofrenia apresentaram alterações cognitivas significativamente mais graves que os diagnosticados como bipolares ou depressivos ${ }^{24}$. Uma meta-análise dos padrões cognitivos de pacientes crônicos com diagnósticos de esquizofrenia, depressão maior e transtorno afetivo bipolar evidenciou maior gravidade das alterações cognitivas na esquizofrenia em relação às outras duas doenças nos diversos aspectos testados $^{3}$. Da mesma forma, as alterações cognitivas da esquizofrenia são mais acentuadas que aquelas do transtorno esquizoafetivo e das psicoses afetivas ${ }^{25}$.

\section{Bases neurobiológicas das alterações cognitivas na esquizofrenia: perspectivas terapêuticas}

Diferentemente do que ocorre nos processos demenciais, onde as alterações cognitivas são consequência da diminuição de atividade e morte neuronais, as alterações cognitivas na esquizofrenia parecem ser consequência de disfunções neuronais ${ }^{26}$. Estudos post mortem não evidenciaram perda neuronal em pacientes esquizofrênicos, embora o volume cerebral esteja reduzido ${ }^{27}$. Estudos de neuroimagem demonstraram que pacientes esquizofrênicos com alterações cognitivas apresentaram redução bilateral do volume do lobo frontal, sem, no entanto, apresentar indicadores de lesões neuronais ${ }^{28}$ ou redução do volume e da densidade neuronal no tálamo e sem se constatar perda neuronal ${ }^{29}$. Outros estudos de neuroimagem evidenciaram associação entre alterações cognitivas e disfunções do sistema límbico e de circuitos neuronais es-

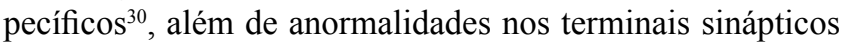
dos neurônios desses circuitos ${ }^{31}$.

Alguns locais têm sido aventados como alvos potenciais para psicofármacos, com o objetivo de melhorar o desempenho cognitivo de pacientes com esquizofrenia em virtude das alterações encontradas: receptores dopaminérgicos no córtex pré-frontal, receptores serotoninérgicos no córtex pré-frontal e no córtex anterior do cíngulo, receptores glutamatérgicos, receptores nicotínicos no hipocampo, receptores muscarínicos, receptores GABAérgicos ${ }^{20}$, além do sistema canabinoide $^{32}$. Vários fármacos têm sido avaliados em modelos animais ${ }^{33}$

\section{Sistema dopaminérgico}

Os receptores D1 estão densamente concentrados no córtex pré-frontal. Estudos de neuroimagem mostraram diminuição da concentração desses receptores no córtex de pacientes esquizofrênicos associada com a gravidade das alterações da memória de trabalho. Agentes agonistas D1 me- 
lhoraram a cognição, enquanto os antagonistas D1 apresentaram efeito oposto ${ }^{34}$. Especificamente, o agonista D1 direto (DAR 100), um inibidor da catecolamina-O-metiltransferase (tolcapone) e um bloqueador da recaptação de noradrenalina (atomoxetina) vêm sendo testados em humanos com fins terapêuticos ${ }^{35,36}$. Entretanto, até o momento, os resultados preliminares com a atomoxetina mostram benefícios limitados na melhora da cognição de pacientes com esquizofrenia ${ }^{36}$. A modafinila é um medicamento utilizado no tratamento da sonolência excessiva diurna. Embora seu mecanismo de ação não esteja bem estabelecido, os sistemas dopaminérgico e noradrenérgico parecem estar envolvidos. Vários pequenos ensaios clínicos foram realizados acerca do emprego dessa substância como adjuvante no tratamento da esquizofrenia, particularmente para sintomas cognitivos e negativos. A heterogeneidade dos estudos e resultados não permitem conclusões acerca da eficácia da modafinila ${ }^{37}$.

Ver também item sobre antipsicóticos, mais adiante.

\section{Sistema serotoninérgico}

Grupos de receptores serotoninérgicos têm sido associados a disfunções cognitivas na esquizofrenia, a saber, 5HT1A, 5HT2A, 5HT3 e 5HT6. Os receptores 5HT1A estão localizados principalmente nos neurônios piramidais do córtex e do hipocampo. Seus antagonistas totais e agonistas parciais parecem melhorar as funções mnésicas ${ }^{34,35}$. Um estudo com a tandospirona sugeriu que essa substância poderia melhorar alguns tipos de funções mnêmicas na esquizofrenia $^{38}$. Entretanto, a buspirona não evidenciou efeitos cognitivos nesses pacientes ${ }^{39,40}$. Os receptores 5HT2A estão localizados principalmente nos neurônios piramidais do córtex pré-frontal e na área tegmentar ventral. $\mathrm{O}$ antagonismo a esses receptores possivelmente pode melhorar o desempenho cognitivo de esquizofrênicos ${ }^{35}$. Os receptores 5HT6 estão concentrados principalmente no córtex olfatório e no hipocampo. Antagonistas seletivos melhoram a memória em modelos animais ${ }^{41}$. Quanto aos receptores 5HT3, a ondansetrona, um antagonista desses receptores, mostrou-se como uma estratégia adjuvante potencial para esquizofrenia crônica em um pequeno ensaio clínico, particularmente para o comprometimento cognitivo e os sintomas negativos ${ }^{42}$.

\section{Sistema glutamatérgico}

O glutamato é o principal neurotransmissor excitatório no cérebro humano, sendo o sistema glutamatérgico altamente complexo e diretamente envolvido com os processos de aprendizado e memória. Sua hiperestimulação está associada a processos neurodegenerativos, incluindo a doença de Alzheimer ${ }^{35}$. As ampaquinas, moduladoras glutamatérgicas, são as principais substâncias em estudo ${ }^{43}$. Dois pequenos ensaios clínicos não mostraram eficácia da memantina como terapêutica adjuvante ao emprego de antipsicóticos para reduzir prejuízos cognitivos em pacientes com esquizofre- nia $^{44,45}$. Entretanto, quando associada à clozapina, foi observada melhora significativa da cognição, avaliada pelo MiniExame do Estado Mental ${ }^{46}$.

\section{Receptores nicotínicos e muscarínicos}

Os receptores nicotínicos estão diminuídos no córtex e no hipocampo de pacientes esquizofrênicos. A acetilcolina tem um importante papel nas funções mnésicas, atentivas e de aprendi$\mathrm{zado}^{34}$. O foco das pesquisas é em agonistas parciais, que, ao contrário da nicotina, não produzem a dessensibilização desses receptores ${ }^{35}$. Os receptores muscarínicos estão diminuídos no córtex pré-frontal de pacientes esquizofrênicos, e a administração de antagonistas muscarínicos piora a capacidade mnésica ${ }^{47}$. Os inibidores de acetilcolinesterase (donepezila, galantamina e rivastigmina), os moduladores muscarínicos (N-desmetilclozapina) e os agonistas muscarínicos (xanomelina) são os principais grupos farmacológicos sob investigação ${ }^{48}$. Os estudos controlados com placebo conduzidos até o momento com os inibidores de acetilcolinesterase não evidenciaram melhora significativa do comprometimento cognitivos nesses pacientes ${ }^{49-51}$. Recentemente, em um pequeno estudo, a xanomelina mostrouse eficaz na melhora do aprendizado verbal e da memória de curto prazo em pacientes esquizofrênicos ${ }^{52}$.

\section{Receptores GABAérgicos}

O aumento dos receptores GABAérgicos (especialmente a subunidade alfa-2) no cérebro de pacientes esquizofrênicos e a comprovação de que moduladores de receptores GABAérgicos alfa-2 melhoram a memória de trabalho nesses pacientes torna esse sistema de neurotransmissão atrativo como alvo de pesquisas ${ }^{34,35}$. Os benzodiazepínicos ativam receptores GABA, alfa-1 e alfa-5, o que piora o desempenho cognitivo, enquanto o flumazenil melhora a cognição, atuando como antagonista desses receptores ${ }^{53}$.

\section{Antipsicóticos}

Vários estudos avaliaram a eficácia desses medicamentos no tratamento das alterações cognitivas da esquizofrenia. Os estudos iniciais indicavam superioridade dos antipsicóticos atípicos em comparação com os típicos ${ }^{54,55}$. Entretanto, estudos posteriores não mostraram superioridade dos atípicos na melhora da cognição dos pacientes com esquizofrenia, seja em pacientes crônicos, seja em pacientes no primeiro episódio ${ }^{56-59}$. Entre os fatores que explicam a diferença de resultados, ressaltam-se o emprego de doses elevadas de antipsicóticos típicos, amostras pequenas e ausência de controle de fatores de confusão, como tratamento anticolinérgico, sintomas extrapiramidais, alteração da sintomatologia positiva e efeito do aprendizado dos testes ${ }^{58}$. Apesar de a clozapina ser considerada mais eficaz no tratamento da esquizofrenia do que os outros antipsicóticos, seus efeitos na cognição não estão bem estabelecidos ${ }^{60-62}$. 


\section{Outras perspectivas terapêuticas}

Além do tratamento psicofarmacológico, os tratamentos psicossociais são fundamentais na abordagem global do paciente com esquizofrenia. Vários programas têm sido desenvolvidos e avaliados para atenuar os déficits cognitivos da esquizofrenia. Esses programas empregam métodos variados, como exercícios práticos, treinamentos realizados em computador, estratégias pedagógicas, estratégias compensatórias e grupos de discussão $0^{63,64}$. A Cognitive Enhancement Therapy (CAT) tem como meta a melhora das alterações cognitivas gerais, e o Errorless Learning tem como objetivo a melhora da cognição social ${ }^{65,66}$. Um programa de treinamento cognitivo foi desenvolvido especificamente para ajudar o paciente com esquizofrenia a se manter empregado ${ }^{63}$. Os resultados sugerem eficácia dessas abordagens ${ }^{63,65}$.

\section{Conclusão}

As alterações cognitivas são a principal causa de incapacidade funcional para o paciente portador de esquizofrenia. Elas têm elevada prevalência e estão presentes mesmo na fase pré-mórbida da doença. Cada vez mais essas alterações são estudadas em diversas áreas, como a neurofisiologia, a genética, a neuropsicologia, a psicologia e a psiquiatria. Com a padronização de instrumentos de avaliação e o avanço das pesquisas, espera-se o surgimento de abordagens terapêuticas eficazes para essas disfunções cognitivas, para permitir uma recuperação mais abrangente dos pacientes com esquizofrenia e, consequentemente, melhorar sua qualidade de vida.

\section{Referências}

1. Razzouk D, Shirakawa I. A evolução dos critérios diagnósticos da esquizofrenia. In: Chaves A, Shirakawa I, Mari JJ, eds. O desafio da esquizofrenia. São Paulo: Editora Lemos; 2001. p. 15-23.

2. Lieberman JA, Drake RE, Sederer LI, Belger A, Keefe R, Perkins D, et al. Science and recovery in schizophrenia. Psychiatr Serv. 2008;59(5):487-96.

3. Krabbendam L, Arts B, van Os J, Aleman A. Cognitive functioning in patients with schizophrenia and bipolar disorder: a quantitative review. Schizophr Res. 2005;80(2-3):137-49.

4. Keefe RS, Fenton WS. How should DSM-V criteria for schizophrenia include cognitive impairment? Schizophr Bull. 2007;33(4):912-20.

5. Ma X, Wang Q, Sham PC, Liu X, Rabe-Hesketh S, Sun X, et al. Neurocognitive deficits in first-episode schizophrenic patients and their first-degree relatives. Am J Med Genet B Neuropsychiatr Genet. 2007;144B(4):407-16.

6. Dickinson D, Ramsey ME, Gold JM. Overlooking the obvious: a meta-analytic comparison of digit symbol coding tasks and other cognitive measures in schizophrenia. Arch Gen Psychiatry. 2007;64(5):532-42.

7. Schelini PW. Teoria das inteligências fluida e cristalizada: início e evolução. Estud Psicol. 2006;11(3):323-32.

8. McGrew KS. Intelligence research \& theory. Part I: Beyond the CHC tipping point: back to the future [Internet] [accessed 2008]. http://www.slideshare.net/ iapsych/beyond-the-chc-tipping-point-back-to-the-future.

9. Sternberg RJ. Inteligência humana e artificial. In: Sternberg RJ. Psicologia cognitiva. Porto Alegre: Artes Médicas; 2000. p. 399-428. Traduzido por MR Borges.
10. Fioravanti M, Carlone O, Vitale B, Cinti ME, Clare L. A meta-analysis of cognitive deficits in adults with a diagnosis of schizophrenia. Neuropsychol Rev. 2005;15(2):73-95.

11. Green MF, Nuechterlein KH. The MATRICS initiative: developing a consensus cognitive battery for clinical trials. Schizophr Res. 2004;72(1):1-3.

12. Green MF, Nuechterlein KH, Gold JM, Barch D, Cohen J, Essock S, et al. Approaching a consensus cognitive battery for clinical trials in schizophrenia: the NIMH-MATRICS conference to select cognitive domains and test criteria. Biol Psychiatry. 2004;56(5):301-7.

13. Buchanan RW, Davis M, Goff D, Green MF, Keefe RS, Leon AC, et al. A summary of the FDA-NIMH-MATRICS workshop on clinical trial design for neurocognitive drugs for schizophrenia. Schizophr Bull. 2005;31(1):5-19.

14. Salgado JV, Carvalhaes CFR, Pires AM, Neves MC, Cruz BF, Cardoso CS, et al. Sensitivity and applicability of the Brazilian version of the Brief Assessment of Cognition in Schizophrenia (BACS). Dement Neuropsychol. 2007;1(3):260-5.

15. Keefe RS, Poe M, Walker TM, Kang JW, Harvey PD. The Schizophrenia Cognition Rating Scale: an interview-based assessment and its relationship to cognition, real-world functioning, and functional capacity. Am J Psychiatry. 2006;163(3):426-32.

16. Zimmer M,Jou GI, Sebastiany CM, Guimarães ER, BoechatLC, Soares T, etal.Avaliação neuropsicológica na esquizofrenia: revisão sistemática [Internet]. Rev Psiquiatr Rio Gd Sul. 2008;30. http://www.scielo.br/scielo.php?script=sci arttext\&pid=S0101-8108200 $8000200005 \& \operatorname{lng}=$ =en. doi: $10.1590 / \mathrm{S} 0101-81082008000200005$

17. Nuechterlein KH, Barch DM, Gold JM, Goldberg TE, Green MF, Heaton RK. Identification of separable cognitive factors in schizophrenia. Schizophr Res. 2004;72(1):29-39.

18. Lee KH, Brown WH, Egleston PN, Green RD, Farrow TF, Hunter MD, et al. A functional magnetic resonance imaging study of social cognition in schizophrenia during an acute episode and after recovery. Am J Psychiatry. 2006;163(11):1926-33.

19. Wilk CM, Gold JM, Humber K, Dickerson F, Fenton WS, Buchanan RW. Brief cognitive assessment in schizophrenia: normative data for the Repeatable Battery for the Assessment of Neuropsychological Status. Schizophr Res. 2004;70(2-3):175-86.

20. Cannon M, Caspi A, Moffitt TE, Harrington H, Taylor A, Murray RM, et al. Evidence for early-childhood, pan-developmental impairment specific to schizophreniform disorder: results from a longitudinal birth cohort. Arch Gen Psychiatry. 2002;59(5):449-56.

21. Rund BR, Melle I, Friis S, Larsen TK, Midbøe LJ, Opjordsmoen S, et al. Neurocognitive dysfunction in first-episode psychosis: correlates with symptoms, premorbid adjustment, and duration of untreated psychosis. Am J Psychiatry. 2004;161(3):466-72.

22. Carpenter WT. Anticipating DSM-V: should psychosis risk become a diagnostic class? Schizophr Bull. 2009;35(5):841-3.

23. Tam WC, Liu Z. Comparison of neurocognition between drug-free patients with schizophrenia and bipolar disorder. J Nerv Ment Dis. 2004;192(7):464-70.

24. Reichenberg A, Harvey PD, Bowie CR, Mojtabai R, Rabinowitz J, Heaton RK, et al. Neuropsychological function and dysfunction in schizophrenia and psychotic affective disorders. Schizophr Bull. 2009;35(5):1022-9.

25. Bora E, Yucel M, Pantelis C. Cognitive functioning in schizophrenia, schizoaffective disorder and affective psychoses: meta-analytic study. $\mathrm{Br} \mathrm{J}$ Psychiatry. 2009;195(6):475-82.

26. Rajkowska G, Selemon LD, Goldman-Rakic PS. Neuronal and glial somal size in the prefrontal cortex: a postmortem morphometric study of schizophrenia and Huntington disease. Arch Gen Psychiatry. 1998;55(3):215-24.

27. Selemon LD, Kleinman JE, Herman MM, Goldman-Rakic PS. Smaller frontal gray matter volume in postmortem schizophrenic brains. Am J Psychiatry. 2002;159(12):1983-991.

28. Roth RM, Flashman LA, Saykin AJ, McAllister TW, Vidaver R. Apathy in schizophrenia: reduced frontal lobe volume and neuropsychological deficits. Am J Psychiatry. 2004;161(1):157-9.

29. Andrews J, Wang L, Csernansky JG, Gado MH, Barch DM. Abnormalities of thalamic activation and cognition in schizophrenia. Am J Psychiatry. 2006;163(3):463-9.

30. Heckers S, Weiss AP, Deckersbach T, Goff DC, Morecraft RJ, Bush G. Anterior cingulate cortex activation during cognitive interference in schizophrenia. Am J Psychiatry. 2004;161(4):707-15.

31. Sawada K, Barr AM, Nakamura M, Arima K, Young CE, Dwork AJ, et al. Hippocampal complexin proteins and cognitive dysfunction in schizophrenia. Arch Gen Psychiatry. 2005;62(3):263-72.

32. Zuardi AW, Crippa JA, Hallak JE, Moreira FA, Guimaraes FS. Cannabidiol, a Cannabis sativa constituent, as an antipsychotic drug. Braz J Med Biol Res. 2006;39(4):421-9. 
33. Marder SR. Drug initiatives to improve cognitive function. J Clin Psychiatry. 2006;67 Suppl 9:31-5; discussion 36-42.

34. Gray JA, Roth BL. Molecular targets for treating cognitive dysfunction in schizophrenia. Schizophr Bull. 2007;33(5):1100-19.

35. Tamminga CA. The neurobiology of cognition in schizophrenia. J Clin Psychiatry. 2006;67(9):e11.

36. Kelly DL, Buchanan RW, Boggs DL, McMahon RP, Dickinson D, Nelson M, et al. A randomized double-blind trial of atomoxetine for cognitive impairments in 32 people with schizophrenia. J Clin Psychiatry. 2009;70(4):518-25.

37. Saavedra-Velez C, Yusim A, Anbarasan D, Lindenmayer JP. Modafinil as an adjunctive treatment of sedation, negative symptoms, and cognition in schizophrenia: a critical review. J Clin Psychiatry. 2009;70(1):104-12.

38. Sumiyoshi T, Matsui M, Nohara S, Yamashita I, Kurachi M, Sumiyoshi C, et al. Enhancement of cognitive performance in schizophrenia by addition of tandospirone to neuroleptic treatment. Am J Psychiatry. 2001;158(10):1722-5.

39. Sumiyoshi T, Park S, Jayathilake K, Roy A, Ertugrul A, Meltzer HY. Effect of buspirone, a serotonin1A partial agonist, on cognitive function in schizophrenia: a randomized, double-blind, placebo-controlled study. Schizophr Res. 2007;95(1-3):158-68.

40. Piskulic D, Olver JS, Maruff P, Norman TR. Treatment of cognitive dysfunction in chronic schizophrenia by augmentation of atypical antipsychotics with buspirone, a partial 5-HT(1A) receptor agonist. Hum Psychopharmacol. 2009;24(6):437-46.

41. Roth BL, Hanizavareh SM, Blum AE. Serotonin receptors represent highly favorable molecular targets for cognitive enhancement in schizophrenia and other disorders. Psychopharmacology (Berl). 2004;174(1):17-24.

42. Akhondzadeh S, Mohammadi N, Noroozian M, Karamghadiri N, Ghoreishi A, Jamshidi AH, et al. Added ondansetron for stable schizophrenia: a double blind, placebo controlled trial. Schizophr Res. 2009;107(2-3):206-12.

43. Javitt DC. Glutamate as a therapeutic target in psychiatric disorders. Mol Psychiatry. 2004;9(11):984-97, 979.

44. Lieberman JA, Papadakis K, Csernansky J, Litman R, Volavka J, Jia XD, et al A randomized, placebo-controlled study of memantine as adjunctive treatment in patients with schizophrenia. Neuropsychopharmacology. 2009;34(5):1322-9.

45. Krivoy A, Weizman A, Laor L, Hellinger N, Zemishlany Z, Fischel T. Addition of memantine to antipsychotic treatment in schizophrenia inpatients with residual symptoms: A preliminary study. Eur Neuropsychopharmacol. 2008;18(2):117-121.

46. de Lucena D, Fernandes BS, Berk M, Dodd S, Medeiros DW, Pedrini M, et al. Improvement of negative and positive symptoms in treatment-refractory schizophrenia: a double-blind, randomized, placebo-controlled trial with memantine as add-on therapy to clozapine. J Clin Psychiatry. 2009;70(10):1416-23.

47. Minzenberg MJ, Poole JH, Benton C, Vinogradov S. Association of anticholinergic load with impairment of complex attention and memory in schizophrenia. Am J Psychiatry. 2004;161(1):116-24.

48. Friedman JI. Cholinergic targets for cognitive enhancement in schizophrenia: focus on cholinesterase inhibitors and muscarinic agonists. Psychopharmacology (Berl). 2004;174(1):45-53.

49. Voss B, Thienel R, Leucht S, Kircher T. [Therapy of cognitive deficits in schizophrenia with acetylcholinesterase inhibitors. A systematic overview]. Nervenarzt. 2008;79(1):47-8, 50-2, 54-9.

50. Keefe RS, Malhotra AK, Meltzer HY, Kane JM, Buchanan RW, Murthy A, et al Efficacy and safety of donepezil in patients with schizophrenia or schizoaffective disorder: significant placebo/practice effects in a 12-week, randomized, doubleblind, placebo-controlled trial. Neuropsychopharmacology. 2008;33(6):1217-28.
51. Dyer MA, Freudenreich O, Culhane MA, Pachas GN, Deckersbach T, Murphy E, et al. High-dose galantamine augmentation inferior to placebo on attention, inhibitory control and working memory performance in nonsmokers with schizophrenia. Schizophr Res. 2008;102(1-3):88-95.

52. Shekhar A, Potter WZ, Lightfoot J, Lienemann J, Dubé S, Mallinckrodt C, et al. Selective muscarinic receptor agonist xanomeline as a novel treatment approach for schizophrenia. Am J Psychiatry. 2008;165(8):1033-9.

53. Menzies L, Ooi C, Kamath S, Suckling J, McKenna P, Fletcher P, et al. Effects of gamma-aminobutyric acid-modulating drugs on working memory and brain function in patients with schizophrenia. Arch Gen Psychiatry. 2007;64(2):156-67.

54. Harvey PD, Rabinowitz J, Eerdekens M, Davidson M. Treatment of cognitive impairment in early psychosis: a comparison of risperidone and haloperidol in a large long-term trial. Am J Psychiatry. 2005;162(10):1888-95.

55. Keefe RS, Silva SG, Perkins DO, Lieberman JA. The effects of atypical antipsychotic drugs on neurocognitive impairment in schizophrenia: a review and meta-analysis. Schizophr Bull. 1999;25(2):201-22.

56. Heinrichs RW. Cognitive improvement in response to antipsychotic drugs: neurocognitive effects of antipsychotic medications in patients with chronic schizophrenia in the CATIE Trial. Arch Gen Psychiatry. 2007;64(6):631-2.

57. Crespo-Facorro B, Rodriguez-Sanchez JM, Perez-Iglesias R, Mata I, Ayesa R, Ramirez-Bonilla M, et al. Neurocognitive effectiveness of haloperidol, risperidone, and olanzapine in first-episode psychosis: a randomized, controlled 1-year follow-up comparison. J Clin Psychiatry. 2009;70(5):717-29.

58. Davidson M, Galderisi S, Weiser M, Werbeloff N, Fleischhacker WW, Keefe RS et al. Cognitive effects of antipsychotic drugs in first-episode schizophrenia and schizophreniform disorder: a randomized, open-label clinical trial (EUFEST). Am J Psychiatry. 2009;166(6):675-82.

59. Wittorf A, Sickinger S, Wiedemann G, Klingberg S. Neurocognitive effects of atypical and conventional antipsychotic drugs in schizophrenia: a naturalistic 6month follow-up study. Arch Clin Neuropsychol. 2008;23(3):271-82.

60. Essali A, Al-Haj Haasan N, Li C, Rathbone J. Clozapine versus typical neuroleptic medication for schizophrenia. Cochrane Database Syst Rev. 2009(1):CD000059.

61. Harvey PD, Sacchetti E, Galluzzo A, Romeo F, Gorini B, Bilder RM, et al. A randomized double-blind comparison of ziprasidone vs. clozapine for cognition in patients with schizophrenia selected for resistance or intolerance to previous treatment. Schizophr Res. 2008;105(1-3):138-43.

62. Krakowski MI, Czobor P, Nolan KA. Atypical antipsychotics, neurocognitive deficits, and aggression in schizophrenic patients. J Clin Psychopharmacol. 2008;28(5):485-93.

63. McGurk SR, Mueser KT, Feldman K, Wolfe R, Pascaris A. Cognitive training for supported employment: 2-3 year outcomes of a randomized controlled trial. Am J Psychiatry. 2007;164(3):437-41

64. Dickinson D, Tenhula W, Morris S, Brown C, Peer J, Spencer K, et al. A randomized, controlled trial of computer-assisted cognitive remediation for schizophrenia. Am J Psychiatry. 2010;167(2):170-80.

65. Kern RS, Green MF, Mitchell S, Kopelowicz A, Mintz J, Liberman RP. Extension of errorless learning for social problem-solving deficits in schizophrenia. Am J Psychiatry. 2005;162(3):513-9.

66. Eack SM, Greenwald DP, Hogarty SS, Cooley SJ, DiBarry AL, Montrose DM, et al. Cognitive enhancement therapy for early-course schizophrenia: effects of a two-year randomized controlled trial. Psychiatr Serv. 2009;60(11):1468-76. 